\title{
Control integrado de Premnotrypes vorax (Hustache) mediante manejo de la población de adultos y control químico en el cultivo de papa
}

\author{
Patricio Gallegos G.*, Germán Avalos P.**
}

\begin{abstract}
RESUMEN
Trabajos sobre la fluctuación de la población de adultos de Premnotrypes vorax indican que ésta es mayor luego de la preparación del suelo y siembra del cultivo de papa. El presente estudio pretende definir si la reducción del número de adultos previa a la siembra, permite cosechar tubérculos con bajo daño. Las localidades seleccionadas por la alta incidencia del insecto fueron tres. Antes de la siembra la población de adultos se eliminó mediante trampas y plantas-cebo de papa, a las que se les aplicó Carbofuran líquido en diferentes oportunidades. El cultivo se instaló cuando se redujo el número de adultos por trampa de alrededor de 80 a menos de 8, en lapsos de captura de 15 días.
\end{abstract}

El análisis del porcentaje de tubérculos con daño indicó no existir diferencias estadísticas $(\mathrm{P}=0.05)$ entre los tratamientos que recibieron o no Carbofuran en las diferentes fechas del cultivo. La disminución de la población de la plaga previa a la siembra no permitió que el insecticida demostrara su capacidad de control. El porcentaje promedio de tubérculos con daño, por localidad, fue 8.0, 4.6 y 2.5, valores bastante bajos aún dentro de un estricto control químico dirigido a las larvas del insecto. Por otra parte, en los sitios donde no se realizó el control previo de adultos ni aplicaciones de Carbofuran al cultivo, el daño fue de 97, 56 y 48\%.

Los resultados encontrados señalan que el método de reducción de la población de adultos de $P$. vorax puede ser una buena alternativa para obtener alta sanidad de los tubérculos.

Palabras claves adicionales: $\quad$ trampas, plantas-cebo, Carbofuran, gusano blanco.

Aceptado para publicación: marzo 22, 1996

* Ing. Agr. M.Sc.

** Egdo. Ing. Agr., Dpto. de Protección Vegetal EESC, INIAP, Casilla 340, Quito, Ecuador. 


\section{SUMMARY}

\section{Integrated Control of Premnotrypes vorax (Hustache) By Managing Adult Population and Chemical Control in the Potato Crop}

Previous studies in Premnotrypes vorax indicate that adult population increases immediately after soil preparation and planting. A trial was conducted in three farming communities of Chimborazo province to test this hypothesis that reducing adult population prior to planting would reduce pest damage in harvested tubers. Adult population changes were monitored through the use of insecticide bait traps and potato bait plants with Carbofuran applied at different times. Fields were planted when the adult population was reduced from already 80 to 8 per trap, or less, in each place, during a period of 15 days.

No statistical significance was found to the percent of tubers damaged between the application of chemical product and adult population compared with adult population reduction alone. This means that the use of insecticide was not necessary.

The average of damaged tubers, for each place, was 8.0,4.6, and 2.5. On the other hand the damage to tubers where there was neither pest adult control nor Carbofuran application was 97, 56, and $48 \%$.

According to the results we think that the adult reduction with this method could be a good alternative to reduce damage to tubers, to improve income to potato growers and moreover to decrease the use of insecticides.

Additional index words: traps, bait plants, Carbofuran, Andean Potato Weevil.

El gusano blanco, Premnotrypes vorax, es la principal plaga en el cultivo de la papa en el Ecuador. En los trabajos de diagnóstico ejecutados por el Proyecto FORTIPAPA se encontró que este insecto puede provocar 48\% o más de tubérculos con daño cuando no se aplican las medidas adecuadas de control, y que las pérdidas de rentabilidad alcanzaron $44 \%$ en Chimborazo y $22 \%$ en Cañar (1). El costo del control para los agricultores de Chimborazo, en relación al costo total de producción, llegó a 7.5\% para la variedad Gabriela y a 21.3\% para la variedad Uvilla (4). 
El alto nivel de daño, las pérdidas elevadas de rentabilidad y los altos costos de control nos exigen desarrollar técnicas eficientes y de menor costo.

Los agricultores que cultivan papa siembran inmediatamente después de la preparación del suelo, momento en el cual se encontró la mayor población de adultos (2). En base a esta información, este estudio tiene como objetivo el determinar si eliminando a estos adultos, previamente a la siembra, se consigue disminuir los daños en los tubérculos a la cosecha.

La eliminación de los adultos de $P$. vorax se realizó mediante trampas y plantas-cebo de papa.

El trabajo se desarrolló con agricultores de las comunidades de Rayoloma, Santa Isabel y Cahuají, ubicadas en la provincia de Chimborazo.

\section{MATERIALES Y MÉTODOS}

En el área de estudio $\left(1,000 \mathrm{~m}^{2}\right)$, luego de la preparación del suelo se distribuyeron cada $10 \mathrm{~m}$ las trampas y se sembraron hileras de papa cada $7 \mathrm{~m}$, para ser utilizadas como plantas-cebo. La cantidad de plantas-cebo corresponde al 10\% del total de plantas próximas a sembrarse.

Las trampas consistieron de porciones de plantas de papa colocadas sobre el suelo cubiertas con un cartón de 40 x $40 \mathrm{~cm}$. En las trampas y plantas-cebo se aplicó Carbofuran líquido al 0.09\%.

La evaluación de la población de la plaga en las trampas se realizó cada 15 días, desde la preparación del suelo o antes hasta 50 días después de la siembra. Luego de cada evaluación se retiraron los adultos capturados.

La siembra del cultivo de papa se efectuó cuando la población de adultos se redujo a menos de 8 por trampa, en lapsos de captura de 15 días, para lo cual se requirió de un período de 60 ó 110 días, según la localidad. En este período de tiempo se renovaron las trampas en 7 oportunidades y en las plantas-cebo se aplicó el insecticida en 4 oportunidades en dos de las localidades y en 5 en la otra. En cada caso la cantidad de insecticida total fue de 0.39 y $0.46 \mathrm{~kg}$ ia/ha.

El cultivo se implantó bajo un diseño de parcela dividida en el que la parcela principal $\left(126 \mathrm{~m}^{2}\right)$ consistió de la aplicación o no de insecticida Carbofuran líquido $(0.09 \%)$ al follaje a la emergencia, las subparcelas $\left(42 \mathrm{~m}^{2}\right)$ consistieron del empleo de Carbofuran granulado $1.25 \mathrm{~kg}$ ia/ha a la siembra y al aporque, y un tratamiento testigo sin insecticida. 
Además, se adicionó un tratamiento satélite denominado testigo referencial, fuera del área del ensayo antes indicado, sometido a libre infestación.

Al momento de la cosecha se evaluó el porcentaje de tubérculos con daño de $P$. vorax y el rendimiento.

\section{RESULTADOS Y DISCUSIÓN}

\section{Fluctuación de la población de adultos.}

La fluctuación de la población de adultos, en las tres localidades, se presenta en la Figura 1.

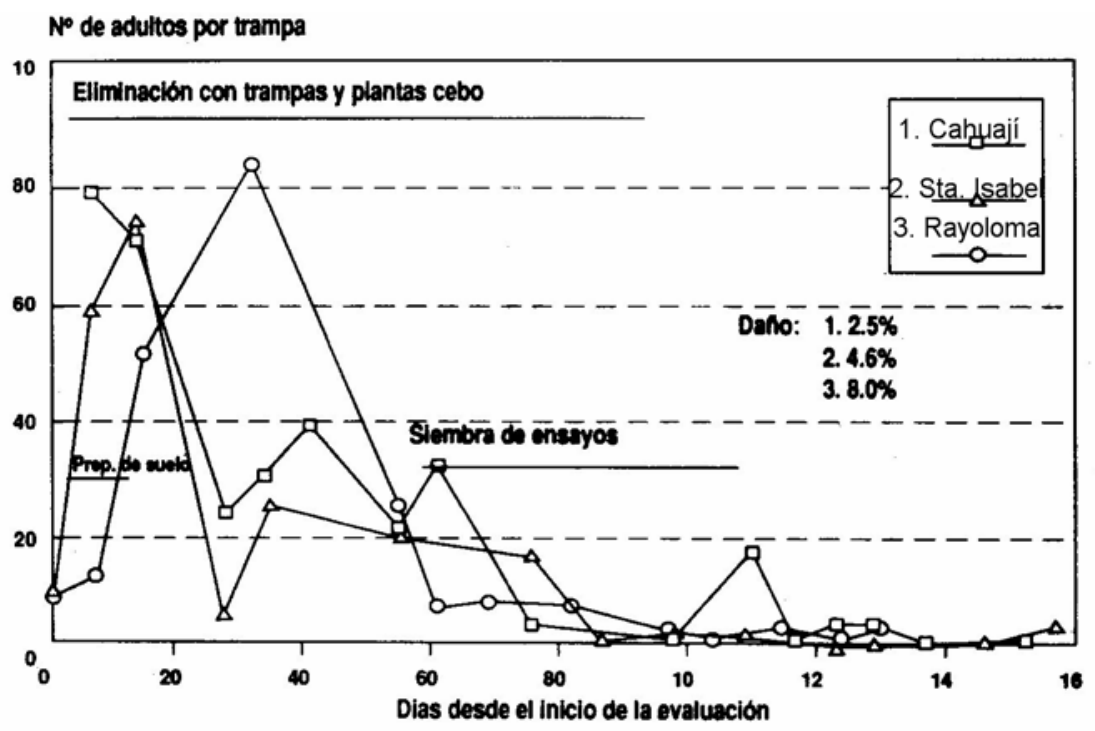

Figura 1. Fluctuación de población de adultos de Premnotrypes vorax en tres localidades. Chimborazo, Ecuador, 1994.

El comportamiento de la población de adultos siguió el mismo patrón en las tres localidades, esto es que luego de la preparación del suelo la densidad de la plaga alcanza su mayor valor, a semejanza de lo encontrado en los trabajos anteriores sobre la fluctuación de la población de este insecto (2). Las capturas promedio más altas fueron de 84 en Rayoloma, 80 en Cahuají y 74 en Santa Isabel.

El empleo de las trampas y plantas-cebo permitió reducir, al momento de la siembra, a 8.4, 3.2 y 3.8 el promedio de adultos por trampa, respectivamente, en cada una de las localidades antes señaladas. Luego de lo cual la plaga se mantuvo en bajos niveles. 
El número total de insectos adultos por hectárea, capturados en las trampas, fue de 33,690 para Rayoloma, 43,040 para Santa Isabel y 49,880 para Cahuají.

Daño de $\boldsymbol{P}$. vorax a los tubérculos: En general el control de la plaga a nivel de agricultor se realiza mediante aplicaciones de insecticidas al suelo y a la base de la planta a fin de afectar a la larva antes de que llegue al tubérculo. Los trabajos de 1992-1993 demostraron que es posible reducir el daño mediante la eliminación de los adultos previamente a la siembra (3). En el ciclo 1993-1994 se realizaron algunos cambios en relación al método del trabajo antes citado, y los resultados se describen a continuación.

El análisis de varianza del porcentaje de tubérculos con daño del insecto indicó no significación estadística para la comparación entre las parcelas principales, esto es entre usar o no Carbofuran líquido al momento de la emergencia. Este resultado se explica por cuanto al haber una población baja de la plaga, por efecto de su eliminación previa, no se manifestaron diferencias entre estos tratamientos.

En la comparación entre los tratamientos de aplicación de Carbofuran granulado a la siembra, al aporque y el tratamiento sin insecticida tampoco se encontró significación estadística, lo que refuerza el hecho de que al no haber insectos el uso del producto químico fue innecesario.

Los promedios de daño en las tres localidades fueron de 8\% en Rayoloma, 4.6\% en Santa Isabel y 2.5\% en Cahuají, los que son bastante bajos, más aún si se considera la alta infestación presente al inicio del trabajo. El daño en los testigos referenciales de 97,56 y 48\% respectivamente, contrasta claramente con los valores del control. Esto sustenta la hipótesis de que la siembra bajo la menor población de adultos puede ser un método para obtener alta sanidad de los tubérculos a la cosecha.

El costo del control para el testigo, en el que únicamente se empleó insecticida para la eliminación de adultos y su respectiva mano de obra, varió entre 55,000 y 65,000 sucres por hectárea. Estas cantidades son muy inferiores al promedio de los agricultores que emplean controles químicos, que según Ramos (4) en Chimborazo llega a 190,000 \$/ha, para la variedad Gabriela (1 US\$=2,400 sucres). En cuanto a la cantidad de Carbofuran empleado de 0.39 y $0.46 \mathrm{~kg}$ de ia/ha en comparación con $1.27 \mathrm{~kg}$ que también reporta Ramos, representa alrededor de 70\% de reducción del tóxico.

El análisis para el rendimiento no mostró significación estadística para tratamiento. En este caso el testigo que no recibió Carbofuran brindó igual rendimiento que los tratamientos que sí lo recibieron. 


\section{CONCLUSIONES}

El método empleado, mediante trampas y plantas-cebo, para reducción de adultos previo a la siembra de papa, demostró que es factible obtener alta sanidad de los tubérculos sin necesidad de recurrir al uso de insecticida al cultivo en sí.

El rendimiento del tratamiento con insecticida no fue inferior estadísticamente a los que sí recibieron producto químico para el control del gusano blanco.

La aplicación del programa de control integrado del gusano blanco permitió alcanzar los objetivos propuestos en esta investigación.

\section{REFERENCIAS BIBLIOGRÁFICAS}

1. Gallegos, P. 1993. Cuantificación de la tecnología utilizada por los productores para el control del gusano blanco de la papa, Premnotrypes vorax. En: Informe Anual Proyecto FORTIPAPA 1992-1993, Subproyecto 3.1.1. Quito, Ecuador.

2. Gallegos, P. 1993. Fluctuación de la población de adultos de Premnotrypes vorax en el cultivo de la papa. Resúmenes del Congreso Latinoamericano de la Papa. Santo Domingo, República Dominicana.

3. Gallegos, P. 1993. Control integrado de Premnotrypes vorax mediante manejo de la población de adultos y control químico en el cultivo de papa. En: Informe Anual Proyecto INIAP-FORTIPAPA 1992-1993. Subproyecto 3.1.6. Quito, Ecuador.

4. Ramos, H; Flores, R.; Salazar, P; Hibon, A. 1993. Determinación de la ventaja comparativa de la producción de papa en el Ecuador, implicaciones para la asignación de recursos de investigación a través de regiones. IDEA. Documento Técnico No. 53. Quito, Ecuador. 